Bryn Mawr College

Scholarship, Research, and Creative Work at Bryn Mawr

College

Graduate School of Social Work and Social

Graduate School of Social Work and Social

Research Faculty Research and Scholarship

Research

2011

\title{
Challenges and solutions for Latin American- trained international medical graduates in psychiatry residency
}

Carolina Hausmann-Stabile

Bryn Mawr College, chausmanns@brynmawr.edu

Luis H. Zayas

David Hauser

Carlos Carvajal

Carlina Mejia

See next page for additional authors

Let us know how access to this document benefits you.

Follow this and additional works at: http://repository.brynmawr.edu/gsswsr_pubs

Part of the Social Work Commons

\section{Custom Citation}

Hausmann-Stabile, C., Zayas, L. H., Hauser, D., Carvajal, C., Mejia, C., Nieves, D. 2011. Challenges and solutions for Latin Americantrained international medical graduates in psychiatry residency. International Journal of Mental Health, 39/40.

This paper is posted at Scholarship, Research, and Creative Work at Bryn Mawr College. http://repository.brynmawr.edu/gsswsr_pubs/83

For more information, please contact repository@brynmawr.edu. 
Authors

Carolina Hausmann-Stabile, Luis H. Zayas, David Hauser, Carlos Carvajal, Carlina Mejia, and Delia Nieves 
International Journal of Mental Health, vol. 40, no. 3, Fall 2011, pp. 29-40. (C) 2011 M.E. Sharpe, Inc. All rights reserved.

ISSN 0020-7411/2011 \$9.50+0.00.

DOI 10.2753/IMH0020-7411400302

CARolina Hausmann-STABile, Luis H. ZaYas, David Hauser, Carlos Carvajal, Carlina Mejia, AND DELIA NIEVES

\title{
Challenges and Solutions for Latin American-Trained International Medical Graduates in Psychiatry Residency
}

\begin{abstract}
Objective: Latin American-trained international medical graduates (IMGs) in psychiatry residency face multiple challenges that go beyond their medical training. These challenges call for innovative problem-solving. Although the professional development of IMGs has been discussed in the professional literature, little is written about their experiences. In this report, a group of IMGs reflect on their experiences and describe how they solved challenges. Method: Using cogenerative ethnography, four IMGs trained in Colombia, the Dominican Republic, and Mexico provided insights on their
\end{abstract}

Carolina Hausmann-Stabile, MA, LMSW, is a National Institute of Mental Health predoctoral fellow at Washington University in St. Louis. Luis H. Zayas, Ph.D., is the Shanti K. Khinduka Distinguished Professor of Social Work and the Director of the Center for Latino Family Research at Washington University in St Louis. David Hauser, M.D., is a psychiatry resident at Lincoln Hospital, New York. Carlos Carvajal, M.D., is a psychiatry resident at Lincoln Hospital, New York. Carlina Mejia, M.D., is a psychiatry resident at Georgetown University Hospital, Washington D.C. Delia Nieves, M.D., is psychiatry resident at Lincoln Hospital, New York.

Support for this article was provided by the Center for Latino Family Research, George Warren Brown School of Social Work, and by a Training Grant from the National Institute of Mental Health of the U.S. Public Health Service (NIMH-T32 MH19960). Correspondence should be sent to C. Hausmann-Stabile, George Warren Brown School of Social Work, Washington University in St. Louis, One Brookings Drive, St. Louis, MO, 63130; e-mail: chausmann@gwbmail.wustl.edu. 
challenges and solutions while adapting to psychiatric residency training. Individual interviews, focused discussion, and written reports were analyzed using data reduction, data display, and conclusion-drawing techniques. Results: We illustrate the challenges of IMG training in psychiatry using their reflections and stories. We categorize these challenges into three domains: immigration and acculturation, social adjustment, and medical training. Quotes were selected to illustrate IMGs' challenges and coping strategies. Conclusion: Some of the combined cultural, social, and academic challenges of Latin American-trained IMGs in psychiatry residency are described. Recognizing and planning for the personal challenges of IMGs in psychiatry can enhance the transition into psychiatric training. Ultimately, improvements in $I M G$ training converts into improved healthcare for all patients.

International medical graduates (IMGs) are a dynamic part of the U.S. healthcare system in general and in psychiatry in particular. IMGs now comprise 25 percent of physicians in the United States [1] and 31.2 percent of all psychiatry residents [2]. The majority of IMGs are recent immigrants to the United States, whereas others are U.S. citizens who received their medical training abroad [3]. IMGs enrich our medical labor force through their diversity, their experiences with diseases less often seen in the United States, and their cultural and linguistic skills that make them vital to immigrant and minority patients. During their postgraduate residency training, they all must adapt to new academic and professional demands; for some this process includes adjusting to a new linguistic, cultural, and social environment.

Academic psychiatry has focused primarily on selecting IMG residents, the supply of IMGs, deficiencies in their training, or their contribution to physician surplus and distribution. Some literature examines the training of IMGs in psychiatry but little focus has been directed toward engaging the diverse talents of IMGs or on helping them balance training demands and develop coping strategies for successfully adjusting to their new circumstances [4]. Some steps have been taken to examine IMGs' experiences during and post training in different medical fields [5-8]. However, little has been done to understand their experiences while transitioning from applicants into their professional psychiatric training. Even less is known about what Latin America-trained IMGs face within and beyond their medical training in psychiatry.

This article enumerates some of the challenges and personal adjustments of IMGs while entering professional training in psychiatry. We describe adjustments made by IMGs and how they turn challenges into advantages. Transitioning from IMG applicant to psychiatry resident is more like navigating an obstacle course than simply moving from Point A to Point B. Some pitfalls can be avoided because they are obvious, but others are not so predictable. 
Uncovering hidden pitfalls brings into sharper focus ways toward smoother, successful transitions. By exploring the experiences of four IMGs training in psychiatry, we group the challenges encountered into categories that lend themselves to intervention by residency programs. We reflect on the successful solutions that helped overcome specific cultural, psychosocial, and academic challenges.

\section{Method}

As part of a course on qualitative research methods, first- and second-year psychiatry residents were encouraged to examine their experiences as IMGs as a path for learning research. The psychiatry residency program is nested in a culturally diverse community-based public hospital in a large metropolis in the northeastern United States. Through a clinical and didactic curriculum centered on an interdisciplinary model and evidence-based approach, the program trains residents to integrate bio-psycho-social theories and techniques to treat a large variety of mental health disorders. As part of the didactic curriculum, the residents are asked to complete two-year-long research classes and immerse themselves in research activities such as generating research protocols, assisting in data collection, analyzing data, and producing scientific reports. The research class participants included four IMGs: a Puerto Rico-born and a U.S.-born Latina who completed their medical training in Dominican Republic; a physician from Colombia; and a white U.S.-born, Mexican-trained doctor. For this project, the class participants played a double role: researchers and research participants. All were bilingual English-Spanish, and their length of stay in the United States, as well as their immigration experiences, educational paths, and levels of acculturation, varied considerably.

Because we had very little empirical knowledge to go on and because we anticipated that the IMGs' views would reflect a complex array of issues related to their experiences while transitioning into their residency training, we determined that an emic approach would be best and would give us an insider's view [9]. Using cogenerative ethnography [10], the class participants and a trained co-researcher examined the IMGs experiences within a cultural, social, and educational context. Cogenerative ethnography is a qualitative method that allows two or more participants to position themselves both as participants and researchers. It attempts to create text that is anchored in narratives of personal experiences, as means for pointing out what is common to the group. In the telling of these personal narratives, the participants talked, wrote, and provided their insights on the challenges of entering and adapting to psychiatric residency training in a large urban medical center. The data collection took place during the class encounters and in collaborative meetings. 
The data were collected using multiple sources including reports written by the IMGs about their immigration and academic experiences, individual interviews, and focused discussions conducted by a trained researcher. The most important data collection technique was "an ongoing dialogue with each other ... [using] unstructured conversation that allowed [us] to ask and answer questions to direct the dialogue and increase understanding" [10, $p$. 585]. The data analysis strategy consisted of three primary steps: data reduction (i.e., identifying themes), data display (i.e., organizing the data in meaningful ways), and conclusion drawing.

\section{Results}

\section{Challenges: Immigration and Acculturation}

Immigration is a daunting personal challenge for most people. As persons make their way in a new country, they often encounter cultural and linguistic barriers. Acculturation involves adapting to a new culture and adopting some of its "apparatus of language, symbols, and interpretations" [11, p. xi] while maintaining elements of the culture of origin. Adaptation to a new way of life poses great challenges, which, in the case of IMGs, adds to the pressures of professional training. Not unlike other immigrants, a male IMG from Colombia noted, "I have been living in the U.S. for six years, and still do not feel at ease here." The Latino foreign-born psychiatry residents described moving from monolingual and culturally less diverse societies to a multilingual and multicultural one.

One solution was to "immerse ourselves in the hosting culture." Most of the acculturation challenges encountered early in the immigration process represented issues that needed to be addressed quickly. IMGs need to build strategies that will allow them to experience multiculturalism while gaining English proficiency. One IMG said, "I chose the cheapest place for living and studying English. It happened to be an Asian community in San Francisco that had a different way of living and speaking compared to the traditional American and Colombian culture."

Sometimes this immersion into the American way of life took the shape of career detours: completing a master's degree, volunteering, and working in low-skill positions (e.g., nanny, doctor's clerk). Some residency program directors were puzzled by these approaches, and, more often than not, residency officials assessed these survival strategies negatively. However, the cultural understanding gained through the participation in such activities prepared the IMGs to better serve patients and to adjust to the training programs.

Language and culture occupy a special place in the assessment and treat- 
ment of psychiatric disorders [11, 12]. For IMGs whose first language is not English, their linguistic deficiencies might increase the risk for misdiagnoses and poor treatment planning and outcomes. "[When caring for patients] you feel weak if you cannot understand what they say, for instance, when they use slang." Therefore, English fluency should be addressed aggressively to avoid potential clinical errors. Because "American accent" classes (i.e., to reduce the IMGs' accent so that patients and colleagues can understand them) are not available during training, residents made use of all resources available to improve their linguistic competence: software, tutors, and classes. Besides increasing their communication effectiveness, English as a second language classes provided the Latino residents with "a compass for American culture."

Psychiatry is unique as a medical field in that the diagnosis and treatment of mental health disorders require a higher level of personal interactions between the physician and the patient. Furthermore, these interactions become a component of the assessment and treatment, as the psychiatrist uses the feelings and cognitions that are activated by the encounter with the patient as clinical tools. An IMG stated: "Not knowing the patient's culture can affect our reactions in the clinical encounter ... because we do not always know what the normality parameters in this cultural context are." Getting to know the cultural and linguistic net in which psychiatric symptoms are anchored requires training that goes beyond residency curricula. "When you are very different from the patient, you can be an expert in understanding psychosis, but you need to understand the cultural and linguistic nuances shaping the presentation of psychotic symptoms." Lacking an understanding of the cultural norms that configure interpersonal interactions can also affect the IMGs' ability to build therapeutic alliances with the patients. "If you have limitations understanding the patient's culture and language, the patient has a difficult time collaborating with you."

In their interactions with patients and when dealing with unfamiliar cultural nuances, IMGs sought the help of cultural brokers, more culturally attuned peers, mentors, and trainers. "Nurses and social workers are always helpful when dealing with cultural issues, because they often have a history of working with the population that is new to the resident." Non-U.S.-born IMGs found that cultural sensitivity trainings, although needed, provide little help when dealing with U.S. patients, especially because they are focused on informing an American audience about non-American cultural issues, not the reverse. However, it is important to highlight that the value of cultural competency training resides in its embrace of "professional flexibility and openness."

Spanish-speaking IMGs noted that because of their bilingualism, supervisors and administrators often expected them to provide cultural partnerships 
with patients with whom they shared a language but who were from different cultures (i.e., Latinos from diverse countries). "All Latinos are not the same." Through their language skills alone, residents have created cultural bridges to effectively care for these patients, deepening their training through their experiences, and filling an increasing service need.

Cultural constructs model time management practices. American and Latino cultures rely in contrasting time orientation patterns, a contrast that becomes evident in the interactions with colleges and patients. Latino IMGs described feeling "less committed to precise dates and schedules" than their American counterparts. While completing their training in the United States, Latino IMGs feel pushed to maximize visits in detriment of time spent with the patients. "The interaction with my patients has changed substantially... . This is one of the things that I have to struggle with as a psychiatrist in training in this country." Another said, "It feels like we are in a factory, not with patients." Because she lack the time that she felt needed to provide mental health care, a Latino IMG complained, "I feel I am becoming colder." IMGs shared feelings that sometimes the time spent with patients is "oriented towards filling forms." One IMG complained: "I feel that my hands are tied, I feel guilty for not having the time to communicate with my patients. I get angry and frustrated. I do not think that my American trained colleagues have this conflict."

The solution for this dilemma was to rely in a "team work approach": "We helped each other [to] try to spend as much quality time with the patient as possible." Talking to peers and supervisors about time management helped sometimes. Occasionally, when feeling rushed, their strategy was to "ignore, ignore, ignore." This approach often implied working overtime to complete the paperwork neglected while prioritizing time with the patients.

\section{Challenge: Social Adaptation}

Moving to a different society imposes the need to adapt to new models of social interrelations and structures, and group organizations. While IMGs looking for training opportunities in the United States seek to enrich their work and educational experiences, they are often unaware of the substantial social changes they encounter, such as losing their extended family support and their social networks.

Medical-training positions in these developed nations, as well as opportunities for medical employment, have proved a strong draw for physicians from many nations. "[In Puerto Rico], there were not enough open positions in psychiatry for me to be able to stay." Income potential and its impact on professional development were other reasons to come to the United States. "One job here provides enough revenue to be able to afford medical books!" 
The interaction between family members is based on values and behavioral systems [13]. Latino families adhere to a traditional model, in which great attention is placed on family ties and interactions, leading to reciprocal rights and duties among relatives. "I spend so much time on the phone talking to my family." For Latino IMGs, these close ties to the family of origin translate sometimes into the need to provide their families with financial support, an experience that increases personal sacrifice during training. This need to support family, in turn, limits IMGs' ability to pursue costly educational opportunities that might heighten their chances for professional success. The financial burdens imposed by family obligations and personal relocation were addressed by "living cheaply" and "working more." Another solution was to "familiarize yourself with the cost of living, as well as with the requirements and characteristics of the areas where you plan to apply" to establish a clear financial plan prior to entering residency.

Most Latino IMGs' families are often unable to offer guidance in dealing with, or even understanding, the challenges faced by the trainees when pursuing a career in the United States. Again and again, their own families' confusion with this process creates additional stress for these residents, increasing their sense of isolation. "Without support from family . . . one should expect to spend much of their time working." This challenge was addressed by building family-like relationships with other IMGs in their training program. These personal ties were described as having a high quality of warmth, support, and closeness. "You become family."

Interpersonal networks are vital for persons' social integration. Sharing social backgrounds and an understanding of social norms allows individuals to access and expand social networks, facilitating mutually supportive relationships. The loss of their social linkages makes Latino IMGs vulnerable to social isolation, identity crises, and anxiety. "It is very hard to make friends from the U.S. when you are a newcomer." Latino IMGs immigrating to the United States to train in residency programs may also experience disruptions in their social status. An IMG might, for example, suddenly find his- or herself a member of a minority group, both among colleagues and in the larger society. "In order to cut down on expenses, one may be forced to live in an undesirable area or in a situation previously unimaginable." With the loss of family support and social networks also comes the loss of the sense of belonging to the community.

IMGs addressed these issues by targeting small, diverse training programs nested in metropolitan areas with large immigrant communities. "You do not stand out being different: everybody here is different." Small programs in which "staff and peers are friendlier" help further the social integration of foreign born IMGs. 


\section{Challenge: Medical Training}

Many of the challenges faced by medical graduates entering and completing a residency program are universal. However, often the challenges encountered by IMGs training in psychiatry are greater or more complex than those faced by U.S. medical graduates (USMGs).

The residency program application process can be puzzling for graduates who are not familiar with the U.S. medical educational system. For instance, because most training programs seek candidates with an American experience and professional references, applications from skillful IMG candidates can languish for months if they do not have strong recommendation letters from the United States. One IMG noted, "Recommendations from foreign countries are not as valued, no matter how prestigious the writer or the institution." Developing professional relationships in the United States that will ultimately lead to positive recommendations of applicants takes time and effort, thus delaying the residency application process. Often, they recalled been asked "why it took you so long to apply?" Psychiatry residency candidates should be aware of these challenges and actively build professional relationships before beginning the application process. A solution is to show continued professional development (e.g., volunteering, medical research) and highlight relevant experience in the country of origin.

In facing the application process, seeking out trainees from similar backgrounds proved useful for some of the IMGs we interviewed. Communicating by phone or e-mail with IMGs currently in training helps to acquire a firsthand account of their experiences and resources. Targeting social networking organizations (e.g., expatriates clubs, community institutions) is helpful; even internet socializing (e.g., blogging, social network sites) can be useful. These support systems "will be invaluable because oftentimes it is the idiosyncratic challenge that can be most vexing."

Literature addressing the application process was an excellent resource for Latin American-trained IMGs. This literature provided valuable step-by-step descriptions of the application process and tips for getting into a residency program. For instance, one of the books addressed how to answer psychiatry residency interview questions regarding the desire to immigrate to America. Vora [14, p. 103] pointed out, "[During interviews] there is always the expectation that you will be going back to your country of origin." Another solution was to look into the vast storehouse of trial-and-error experiences available in the blogosphere.

A major challenge for most IMGs who do not have American citizenship is the lack of financial support during the residence application process. For instance, California, while perhaps a very desirable place to live, was not 
seen by our group as "IMG friendly." "The process in California is more difficult as you need to obtain a costly screening letter in order to verify additional applicant information." "A candidate may have a significantly greater chance of obtaining a residency in an East Coast city like NYC." Applying in multicultural urban areas has a double benefit: First, it increases the chance of being selected due to the need of multicultural physicians, and, second, IMGs might find themselves living in a place where cultural, racial, and ethnic minorities thrive.

Finally, participants found that attending every residency interview, although challenging, costly, and stressful, paid off. "I didn't want to go to the Bronx, and at the end I took the chance, and I discovered a great world. . . . Nobody knows about the Bronx. ... Why go to the Bronx to study? It was a great door for opportunities."

Additionally, IMGs are often not as familiar with medical technology as USMGs because their education may not have included advanced technologies such as CT or MRI imagery. Some IMGs reported feeling that their educational background was more focused on the clinical aspects of psychiatric care (e.g., diagnosing, treating), and not so much in the procedures for mental health care. "In the U.S., there is a protocol and schema to follow in everything you do." Physicians in the United States are accustomed to working in the context of multiprofessional teams, and patients are encouraged to express their opinions and to educate themselves about health issues. In Latin America, physicians are often the only professionals that can make decisions about the patient's care, while expecting great deference in exchange for their professional advice. "The patients here have no respect for the doctors!" The ethical, legal, and business aspects of the medical profession, with which most U.S. medical graduates are familiar, were puzzling to this group of IMGs. "I had to relearn what was legal and what not, the names of the medications ... and all the insurances." Again, mentors who could help identify the residents' strengths and weakness in their relationship with colleges, team members, and patients were crucial to improving the IMGs learning experiences and helped further understanding of the norms and standards of medicine as practiced in the United States.

Every student encounters tests in medical school, but most IMGs reported struggling with standardized testing. In the United States, knowledge assessment relies heavily on multiple-choice exams, and students are exposed to this kind of testing beginning in elementary school. Overseas, oral, bedside clinical assessments, and written essay exams prevail [15, p. 128]. Latino IMGs reported that the standardized examination system is challenging. They cited their lack of experience with the testing method as well as difficult content and linguistic nuances. Additionally, U.S. medical schools focus pre- 
dominantly on U.S. board exam preparation whereas IMGs are not exposed to this type of exam-specific training. A learning-for-the-exam orientation is a very different model. Further, implicit in that model is a focus on diseases that are most commonly encountered in the United States, which may be all but unheard of in foreign countries.

At the time of their residency application, most IMGs are older and often have a greater number of years since graduation from medical school than many USMGs [1]. Although their years of experience practicing medicine in Latin America helped them with the clinical aspects of the U.S. Medical Licensing Examinations (USMLEs), one IMG shared, "I have been out of school for years.... My knowledge of general medicine and basic sciences is not as fresh." Success in these examinations is often linked to work contracts, a critical factor in continuing to receive a work visa. Therefore, favorable outcomes in the USMLEs are tied not only to their training continuity but, ultimately, to their chances of remaining in the United States.

Latin American-trained IMGs often solved this challenge by seeing the first round of USMLEs as an expensive practice. "You have to learn to take the test." In this way, IMGs also allowed themselves enough time to retake the exams without jeopardizing their residency contracts. In addition, residents sought the advice of mentors and peers, including fellow IMGs who had successfully completed their certifications and USMGs who could provide an inside perspective of the process. Practicing exam taking, although time-consuming, helped residents to become familiar with the standardized tests. "Studying time during residency is very limited, as the demands of a city hospital with an expanding patient population put an added burden on the training physician" was a common experience articulated by the residents. Support and mentorship from colleagues and faculty was critical to the residents' ability to manage the stress related to the tests.

Because of the recent demographic changes in the United States [16], bilingual Spanish-English psychiatry residents are in high demand. While training in community-based health care organizations based in areas with large concentrations of Latinos, the Latin American-trained residents find themselves very early in their training, caring for large case loads. "In residency psychiatry training, we have a higher number of patients in our case loads. Much earlier than other residents, and with much more patients than in other trainings."

\section{Conclusion}

Latin American-trained IMGs in psychiatry residency identified a wide range of challenges and solutions. Whereas previous studies use administrative or 
survey data [4], this project drew on reported experiences by a small group of IMGs. Some of these issues are often invisible to academic psychiatry, as well as to the organizations that deliver the psychiatrist's training. We recognized that the IMG population is more diverse than the group presented here and that our sample size and characteristics limit the generalizability of our findings. However, we hope to take a small step to address the dearth of literature describing the experiences of Latin American-trained IMGs in psychiatry and to encourage the further development of empirical research about the broader issues faced by this group.

Learning to be a physician is a process that goes beyond medical training and education. All Latin American-trained IMGs pursuing psychiatry training in the United States must learn the professional and academic aspects of their profession. Some must also gain linguistic, cultural, and social competence. Support from peers, colleagues, and mentors may be especially useful for IMGs who may struggle with aspects of the host country or training program.

Identifying challenges faced by IMGs and offering solutions may go some length in providing "preventative medicine" to both future IMGs and residency programs alike. Prior knowledge of the issues mentioned in this article would have saved the Latin American-trained IMGs lost time and money, while reducing stress and enhancing their quality of life. Residency programs will benefit by ameliorating predicable pitfalls and utilizing the strengths of IMGs that these challenges often overshadow.

\section{References}

1. Akl, E.A.; Mustafa, R.; Bdair, F.; \& Schünemann, H.J. (2007) The United States physician workforce and International Medical Graduates: Trends and characteristics. Journal of General Internal Medicine, 22(2), 264-268.

2. Halles, D.J.; Delanoche, N.; \& Cope, C. (2007) Residents census: Characteristics and distribution of psychiatry residents in the U.S., 2006-2007. Arlington, VA: American Psychiatric Association. Available at www.psych.org/MainMenu/ EducationCareerDevelopment/EducationalInitiatives/residentcensus/0910census. aspx?FT=.pdf, accessed February 9, 2011.

3. Johnson, A.; Hagopian, A.; Veninga, C.; Fordyce, M.A.; \& Hart, L.G. (2005) The changing geography of American graduating from foreign medical. WWAMI Center for Health Workforce Studies working paper no. 96.

4. Rao, N.R.; Kramer, M.; Saunders, R.; Twemlow, S.; Lomax, J.; Dewan, M.J.; et al. (2007) An annotated bibliography of professional literature on International Medical Graduates. Academic Psychiatry, 31(1), 68-83.

5. Klein, D.; Hofmeister, M.; Lockyer, J.; Crutcher, R.; \& Fidler, H. (2009) Push, pull, and plant: The personal side of physician immigration to Alberta, Canada. Family Medicine, 41(3), 197-201.

6. Fiscella, K.; Roman-Diaz, M.; Lue, B.H.; Botelho, R.; \& Frankel, R. (1997) 
"Being a foreigner, I may be punished if I make a small mistake": Assessing transcultural experiences in caring for patients. Family Practice, 14(2), 112-116.

7. McMahon, G.T. (2004) Coming to America: International medical graduates in the United States. New England Journal of Medicine, 350(24), 2435-2437.

8. Khorana, A.A. (2008) Disorientation. Health Affairs, 27(4), 1154-1159.

9. Morse, J.M. (2003) Principles of mixed methods and multi-method research design. In A. Tashakkori \& C. Teddlie (Eds.), Handbook of mixed methods in social and behavioral research (pp. 189-208). Thousand Oaks, CA: Sage.

10. Grenier, R.S., \& Burke, M.C. (2008) No margin for error: A study of two women balancing motherhood and Ph.D. studies. Qualitative Report, 13(4), 581-604.

11. Kleiman A. (1991) Rethinking psychiatry. From cultural category to personal experience. New York: Free Press.

12. Zayas, L.H.; Torres, L.R.; \& Cabassa, L.J. (2009) Diagnostic, symptom, and functional assessments of Hispanic outpatients in community health practice. Community Mental Health Journal, 45(2), 97-105.

13. Keefe, S.E. (1984) Real and ideal extended familism among Mexican Americans and Anglo Americans: On the meaning of as "close" family ties. Human Organization 43(1), 65-70.

14. Vora, A.A. (2005) The successful IMG: Obtaining a U.S. residency. Malden, MA: Lippincott Williams \& Wilkins.

15. Rao, N.R. (1999) International medical graduates. In J. Kay, E.K. Silberman, \& L. Pessar (Eds.), Handbook of psychiatric education and faculty development (pp. 125-141). Washington, DC: American Psychiatric Association.

16. U.S. Census Bureau (2004). U.S. interim projections by age, sex, race, and Hispanic origin, 2000-2050. Washington, DC. Available at www.census.gov/population/www/projections/usinterimproj, accessed February 15, 2011. 
Copyright of International Journal of Mental Health is the property of M.E. Sharpe Inc. and its content may not be copied or emailed to multiple sites or posted to a listserv without the copyright holder's express written permission. However, users may print, download, or email articles for individual use. 